\title{
Anti-inflammatory effects of triptolide by inhibiting the NF-кB signalling pathway in LPS-induced acute lung injury in a murine model
}

\author{
XIAN WANG ${ }^{1,2}$, LEI ZHANG ${ }^{3}$, WEI DUAN ${ }^{3}$, BIN LIU $^{4}$, PING GONG $^{5}$, YUSONG DING $^{6}$ and XIONGWEN WU ${ }^{1}$ \\ ${ }^{1}$ Department of Immunology, Tongji Medical College, Huazhong University of Science and Technology, Wuhan, \\ Hubei 430000; ${ }^{2}$ Department of Pathogenic Biology and Immunology; Departments of ${ }^{3}$ Clinical Laboratory, \\ ${ }^{4}$ Emergency and ${ }^{5}$ Medical Oncology, The First Affiliated Hospital; ${ }^{6}$ Department of Public Health, \\ College of Medicine, Shihezi University, Shihezi, Xinjiang 832000, P.R. China
}

Received July 30, 2013; Accepted March 25, 2014

DOI: $10.3892 / \mathrm{mmr} .2014 .2191$

\begin{abstract}
Triptolide is one of the main active components in the Chinese herb Tripterygium wilfordii Hook F, which has been demonstrated to possess anti-inflammatory properties. The aim of this study was to investigate the effects of triptolide on lipopolysaccharide (LPS)-induced acute lung injury (ALI) in mice and to explore the possible mechanisms. Mice were administered LPS intranasally to induce lung injury, and triptolide was administered intraperitoneally $1 \mathrm{~h}$ prior to the LPS challenge. Triptolide-treated mice exhibited significantly reduced levels of leukocytes, myeloperoxidase activity and edema of the lung, as well as tumour necrosis factor- $\alpha$, interleukin (IL)-1 $\beta$ and IL- 6 production in the bronchoalveolar lavage fluid compared with LPS-treated mice. Additionally, western blot analysis showed that triptolide inhibited the LPS-induced phosphorylation of nuclear factor of $\kappa$ light polypeptide gene enhancer in B cells inhibitor- $\alpha$ and nuclear factor $\kappa$-light-chain-enhancer of activated B cells-p65 $(\mathrm{NF}-\kappa \mathrm{B}$ p65) and the expression of Toll-like receptor 4 (TLR4). In conclusion, the results from the present study suggest that the anti-inflammatory effect of triptolide against LPS-induced ALI may be due to its ability to inhibit the TLR4-mediated NF- $\mathrm{BB}$ signalling pathway. Triptolide may therefore be a promising potential therapeutic agent for ALI treatment, which may ultimately aid the clinical therapy for patients with ALI.
\end{abstract}

Correspondence to: Dr Xiongwen Wu, Department of Immunology, Tongji Medical College, Huazhong University of Science and Technology, 13 Hangkong Road, Wuhan, Hubei 430000, P.R. China E-mail: wuxiongwentry@163.com

Key words: triptolide, acute lung injury, inflammatory cytokines, Toll-like receptor 4

\section{Introduction}

Acute lung injury (ALI) is a lung disease characterised by severe inflammation of endothelial and epithelial cells of the lung (1). Hypoxemia, pulmonary edema and widespread capillary leakage are usually observed in patients with ALI (2). This is caused by inflammation-related stimuli, primarily sepsis and pneumonia, which lead to leukocyte migration and overproduction of pro-inflammatory mediators, including interleukin (IL)-6, IL-8 and tumour necrosis factor- $\alpha$ (TNF- $\alpha$ ) (3). ALI is a fatal disease that can cause persistent respiratory failure and multiorgan dysfunction, and patients with ALI may be dependent on mechanical ventilation (4-6). Although intensive care has been greatly improved, the mortality rate caused by ALI is between 40 and 70\%, and 200,000 individuals are affected by the condition in America every year. Thus, novel drugs and therapies are required for the treatment of ALI.

Toll-like receptor 4 (TLR4) is the ligand of lipopolysaccharide (LPS), which is one of the pathogen-associated molecular pattern recognition molecules $(7,8)$. TLR4 recognises pathogen-associated proteins, such as LPS, and activates the nuclear factor $\kappa$-light-chain-enhancer of activated $\mathrm{B}$ cells $(\mathrm{NF}-\kappa \mathrm{B})$ signalling pathway, leading to the secretion of inflammatory cytokines and chemokines (9). In chronic diseases, NF- $\kappa \mathrm{B}$ is known to be activated; this efficiently initiates and accelerates the inflammatory response and induces the immune response (10-12). Although it is well established that TLR4 affects downstream NF- $\kappa$ B activation, the effect of triptolide on TLR4-NF- $\kappa \mathrm{B}$ signalling has yet to be elucidated.

Triptolide is a diterpenoid epoxide extracted from the perennial plant Tripterygium wilfordii Hook F, which is known in Chinese as 'lei gong teng' $(13,14)$, and has been used for the therapy of arthritic ailments in Traditional Chinese Medicine for numerous years. $T$. wilfordii contains $>100$ bioactive compounds and triptolide is one of the major biologically active components. In vivo and in vitro experiments have demonstrated that triptolide has a role in numerous immune disorders, including multiple sclerosis, colitis, lupus nephritis and transplant rejection $(6,15,16)$. The anti-inflammatory activity of triptolide not only activates immune cells, such as T cells and monocytes, 
but also resident tissue cells $(17,18)$. In the present study, the anti-inflammatory effects of triptolide were investigated, and the mechanisms underlying the action of triptolide in ALI were explored. This may provide effective treatments for ALI, which may ultimately aid the clinical therapy for patients with ALI.

\section{Materials and methods}

Animals and reagents. Six- to eight-week-old male BALB/c mice were obtained from the Laboratory Animal Center of Shihezi University (Shihezi, China) and housed in specific pathogen-free conditions. All procedures were in accordance with the Declaration of Helsinki of the World Medical Association. The protocols were also approved by the Institutional Animal Care and Use Committee of the Shihezi University School of Medicine. Triptolide was purchased from Shanghai DND Pharm-Technology Co., Inc. (Shanghai, China). The purity of triptolide was $\geq 98 \%$, and the triptolide was dissolved in dimethyl sulphoxide (DMSO). Dulbecco's modified Eagle's medium and fetal bovine serum were provided by Gibco-BRL (Carlsbad, CA, USA). Penicillin, streptomycin and LPS were purchased from Sigma (St. Louis, MO, USA). Antibodies against TLR4, phosphorylated-nuclear factor of $\kappa$ light polypeptide gene enhancer in $B$ cells inhibitor- $\alpha(p-I \kappa B-\alpha)$ and phosphorylated-NF- $\kappa$ B (p65) were purchased from Santa Cruz Biotechnology, Inc. (Santa Cruz, CA, USA), and antibodies against $\beta$-actin were purchased from TransGenic Biotechnology (Beijing, China). Monocyte chemotactic protein (MCP)-1 was purchased from Thermo Fisher Scientific, Inc. (Rockford, IL, USA).

Cytokine determination. For the ALI model, mice were fasted overnight but allowed water ad libitum. Mice were anaesthetised with intraperitoneal pentobarbital $(50 \mathrm{mg} / \mathrm{kg})$, and a dose of $1 \mathrm{mg} / \mathrm{kg}$ LPS was instilled intratracheally to induce ALI. The bronchoalveolar lavage fluid (BALF) was harvested as previously described (19) and the concentrations of inflammatory cytokines (TNF- $\alpha$, IL-1 $\beta$, IL-6 and IL-8) were determined using ELISA kits (Neobioscience, Beijing, China) in accordance with the manufacturer's instructions, using a Benchmark Microplate Reader (Bio-Rad, Hercules, CA, USA). All of the samples were analysed for cytokine levels in duplicate.

Grouping of mice. The mice were randomly divided into six groups, with each group containing $>10$ mice. The negative control and triptolide groups were administered an intravenous injection of corresponding concentrations of DMSO or triptolide, respectively, and after $30 \mathrm{~min}$ they were intratracheally treated with phosphate-buffered saline. The LPS group was treated with an intravenous injection of vehicle DMSO and, after $30 \mathrm{~min}$, received an intratracheal injection of LPS $(1 \mathrm{mg} / \mathrm{kg})$. The LPS + triptolide groups were administered an intravenous injection of triptolide $(1,10$ and $50 \mu \mathrm{g} / \mathrm{kg})$ and, after $30 \mathrm{~min}$, received an intratracheal injection of LPS ( $1 \mathrm{mg} / \mathrm{kg}$ ). Twelve hours after LPS administration, all animals were sacrificed.

Quantitative polymerase chain reaction ( $q P C R)$ analysis of $m R N A$ expression. Total RNA was extracted using a RNApure kit (BioTeke Corp., Beijing, China) and reverse transcribed using murine leukaemia virus-reverse transcriptase (Invitrogen Life Technologies, Carlsbad, CA, USA). The quality of the mRNA was analysed using denaturing agarose gel electrophoresis containing $1.5 \%$ formaldehyde. Each cDNA sample was made in triplicate. PCR amplifications were performed using an Applied Biosystems 7500 Real-Time PCR system (Applied Biosystems $^{\mathrm{TM}}$, Foster City, CA, USA). PCR was performed under the following thermal cycling conditions: 40 cycles of $10 \mathrm{sec}$ at $95^{\circ} \mathrm{C}$ and $1 \mathrm{~min}$ at $60^{\circ} \mathrm{C}$ using SYBR-Green. Primers for macrophage inflammatory protein (MIP)-1 $\alpha$, MIP-1 $\beta$, chemokine (C-C motif) ligand 5 (RANTES), interferon $\gamma$-induced protein-10 (IP-10), MCP-1 and $\beta$-actin were as follows: MIP-1 $\alpha, 5^{\prime}$-TGTTTGCTGCCAAGTAGCCACATC-3' and 5'-AACAGTGTGACCAACTGGGAGGGA-3'; MIP-1 $\beta$, 5'-AAACCTAACCCCGAGCAACA-3' and 5'-CCATTGGTG CTGAGAACCCT-3'; RANTES, 5'-TCGTGCCCACGT CAAGGAGTATTT-3' and 5'-ACTAGAGCAAGCGATGAC AGGGAA-3'; IP-10, 5'-GGTCTGAGTGGGACTCAAGG-3' and 5'-TCTTTTTCATCGTGGCAATG-3'; MCP-1, 5'-TGC ATCTGCCCTAAGGTCTTC-3' and 5'-AAGTGCTTGAGG TGGTTGTGG-3'; $\beta$-actin, 5'-AGAGGGAAATCGTGC GTGAC-3' and 5'-CAATAGTGATGACCTGGCCGT-3'.

Myeloperoxidase (MPO) assays. Tissue samples from different groups of mice were subjected to four freeze-thaw cycles and centrifuged at $12,000 \mathrm{x}$ f for $10 \mathrm{~min}$ at $4^{\circ} \mathrm{C}$. The supernatant was assayed for MPO activity using ELISA kits of MPO (R\&D Systems Inc. Minneapolis, MN, USA) in accordance with the manufacturer's instructions.

Lung wet/dry weight ratio. The wet/dry weight ratio is an indicator of lung edema. The ratios were calculated by dividing the wet weight by the dry weight. The middle lobe of the right lung was excised and the wet weight was recorded, and the lung was then placed in an incubator at $80^{\circ} \mathrm{C}$ for $24 \mathrm{~h}$ to obtain the dry weight.

Pulmonary histopathology. The lobe of the left lung was harvested $12 \mathrm{~h}$ after LPS administration and fixed with an intratracheal instillation of $1 \mathrm{ml}$ buffered formalin $(10 \%$, $\mathrm{pH}$ 7.2). The following procedures were performed as previously described $(20,21)$. The lobe was further fixed in $10 \%$ neutral buffered formalin for $24 \mathrm{~h}$ at $4^{\circ} \mathrm{C}$. The tissues were embedded in paraffin and cut into $5-\mu \mathrm{m}$ sections. Hematoxylin and eosin staining was performed following the standard protocol.

Western blot analysis. Western blot assay was performed according to the methods of Lewis et al (22) and VallejoIllarramendi et al (23). Briefly, A549 cells were seeded into 48-well plates. After $6 \mathrm{~h}$, the adherent cells were treated with $100 \mathrm{ng} / \mathrm{ml}$ LPS along with various concentrations of triptolide for $1 \mathrm{~h}$. The total cell lysates were prepared. The expression levels of TLR4, p-IкB- $\alpha, \mathrm{p}-\mathrm{NF}-\kappa \mathrm{B}$ (p65) was detected and $\beta$-actin and lamin $\mathrm{B}$ were used as controls. Briefly, the steps included gel electrophoresis, transfer, blocking, incubation with primary antibody and secondary antibody, colorimetric detection. Antibodies against TLR4, p-I $\kappa$ B- $\alpha$ and $p-N F-\kappa B$ (p65) were purchased from Santa Cruz Biotechnology, Inc. and 


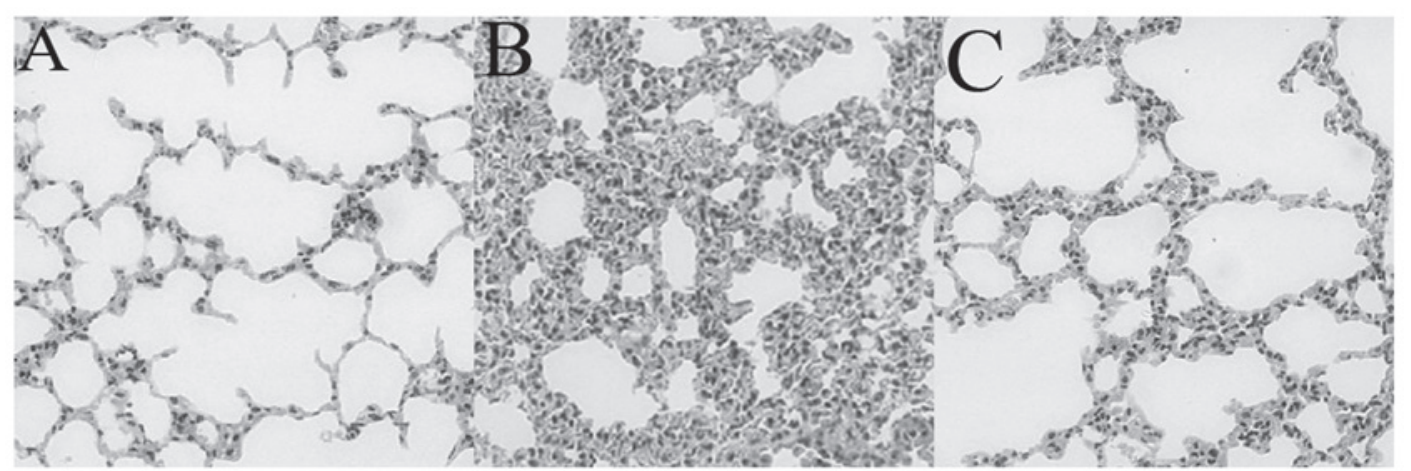

Figure 1. Pulmonary histopathological alterations in mice in the (A) normal, (B) LPS and (C) LPS + triptolide groups. Lung sections were stained with hematoxylin and eosin $8 \mathrm{~h}$ after LPS administration to reveal histopathological changes (magnification, x200). Compared with the (A) normal group, the (B) LPS group exhibited a thickened alveolar wall and notable inflammatory cell infiltration. (C) The LPS + triptolide group exhibited marked histopathological differences to the LPS group, with fewer infiltrated inflammatory cells and a thinner alveolar wall. LPS, lipopolysaccharide.

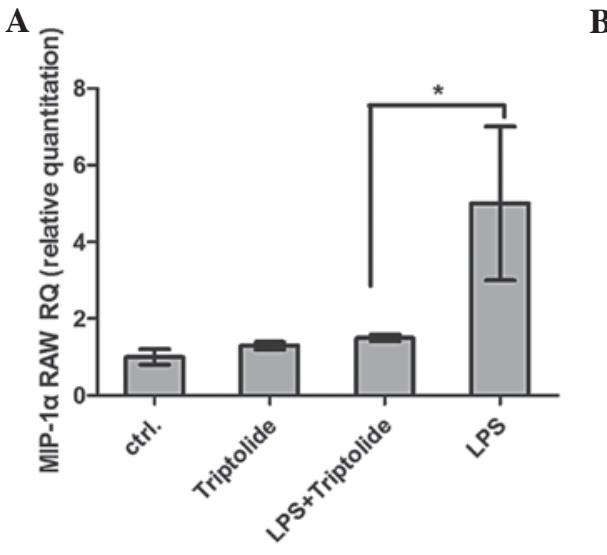

D
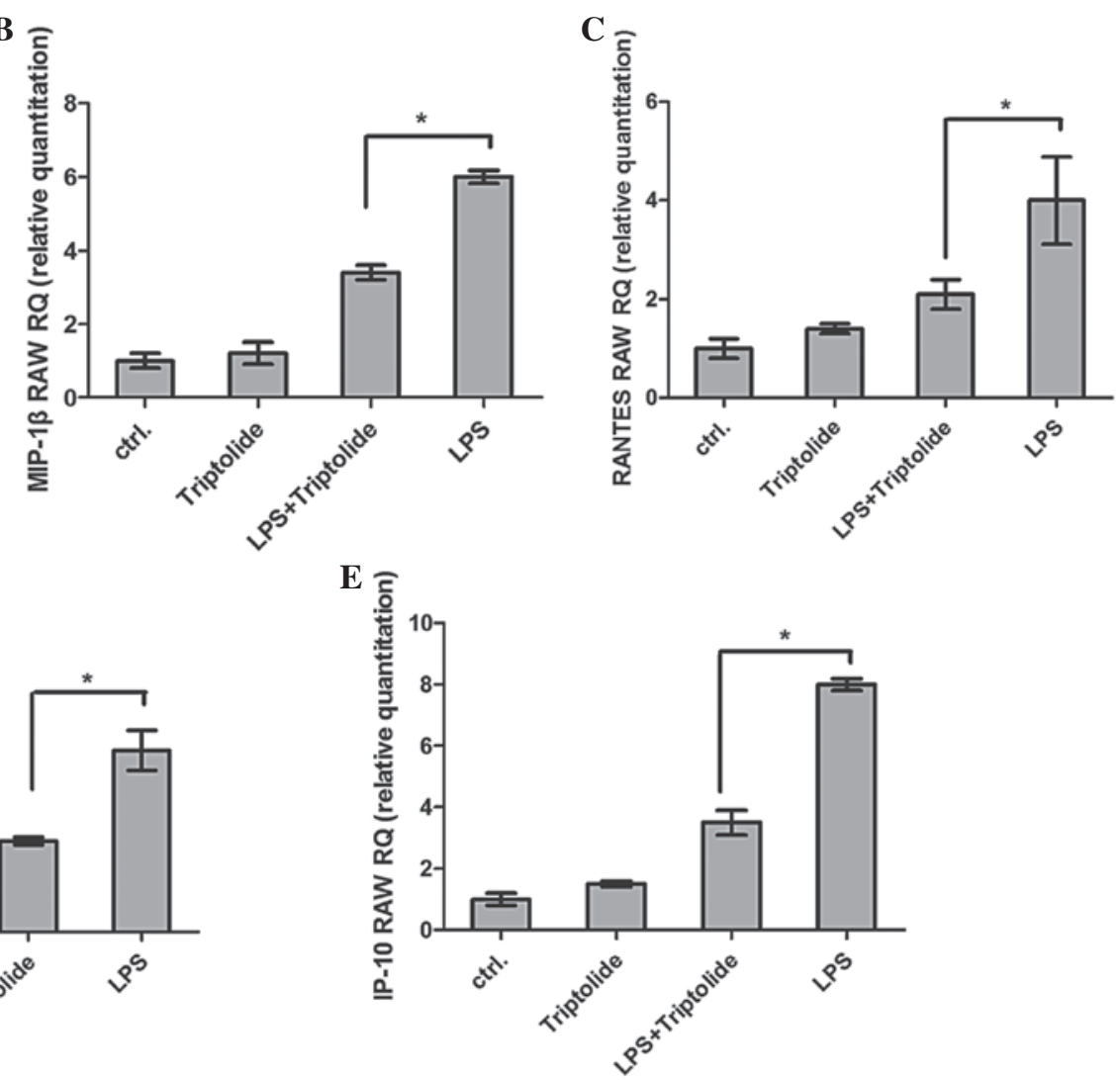

Figure 2. Effect of triptolide on the expression of chemokines in lung tissues of mice. Mice treated with triptolide $(50 \mu \mathrm{g} / \mathrm{kg})$ showed significantly reduced expression levels of (A) MIP-1 $\alpha$, (B) MIP-1 $\beta$, (C) RANTES, (D) MCP-1 and (E) IP-10 following LPS administration. Data are presented as the mean \pm standard deviation; " $\mathrm{P}<0.05$ vs. LPS group. LPS, lipopolysaccharide; MIP, macrophage inflammatory protein; MCP-1; monocyte chemotactic protein-1; IP-10, interferon $\gamma$-induced protein-10; RANTES, chemokine (C-C motif) ligand 5; ctrl, negative control.

antibodies against $\beta$-actin were purchased from TransGenic Biotechnology (Beijing, China). The secondary antibodies of goat anti-mouse IgG and goat anti-rabbit antibody were obtained from Santa Cruz Biotechnology, Inc.

Statistical analysis. Data were entered into a database and analysed using SPSS statistical software (SPSS, Inc., Chicago, IL, USA). Data are expressed as the mean \pm standard deviation or the standard error of the mean. Analysis of variance was used to determine statistically significant differences between groups, followed by the Student's t-test. $\mathrm{P}<0.05$ was considered to indicate a statistically significant difference.

\section{Results}

Triptolide improves the pulmonary histopathological status of mice with ALI. The pulmonary histopathological alterations of the mice are shown in Fig. 1. In the LPS group, the lung tissues stained with hematoxylin and eosin exhibited widespread alveolar wall thickness, alveolar collapse and notable inflammatory 
A

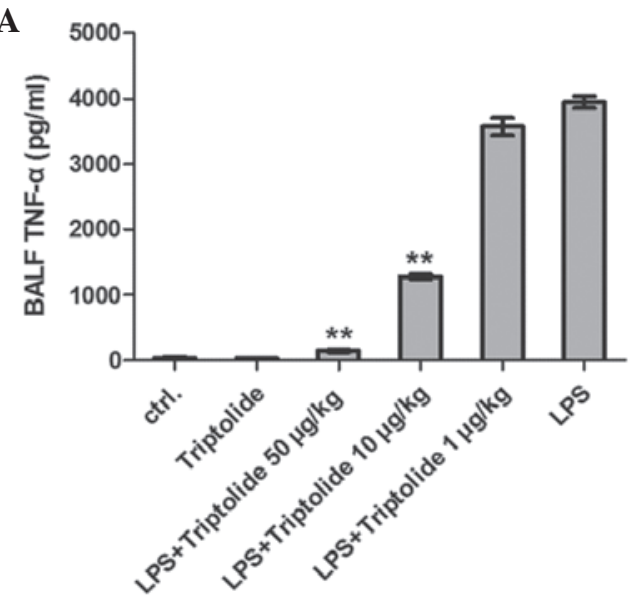

C

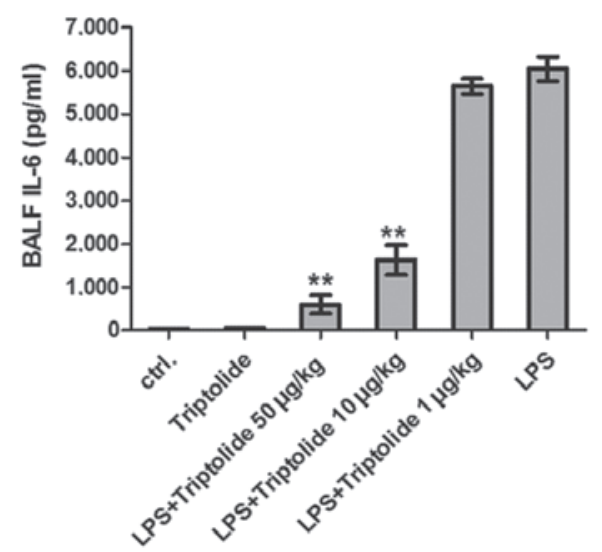

B

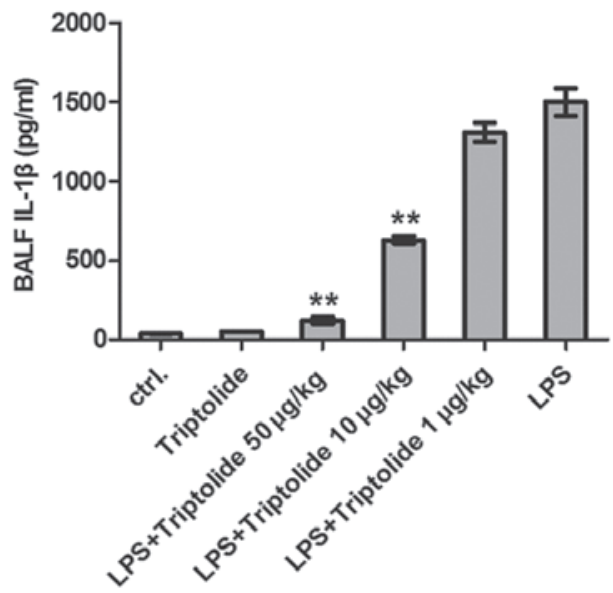

D

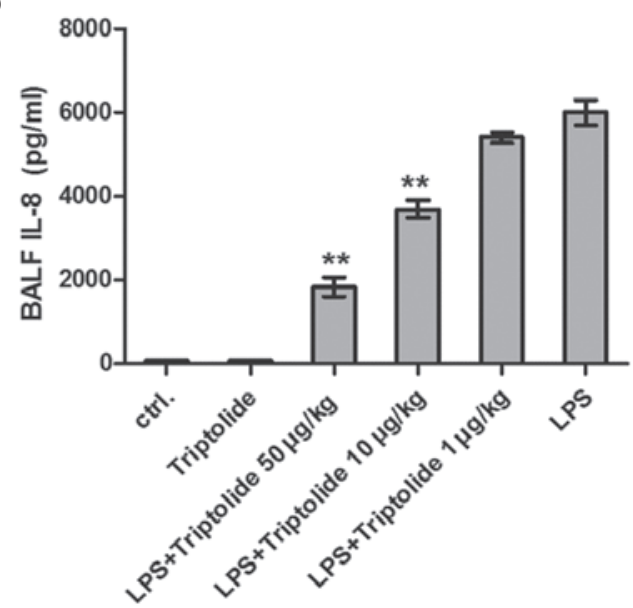

Figure 3. Cytokine production in BALF treated with triptolide. Mice were treated with LPS $(1 \mathrm{mg} / \mathrm{kg})$ with different concentrations of triptolide $(1,10$ and $50 \mu \mathrm{g} / \mathrm{kg}$ ) for $8 \mathrm{~h}$. The concentrations of (A) TNF- $\alpha$, (B) IL-1 $\beta$, (C) IL-6 and (D) IL-8 in the BALF following LPS administration were measured using ELISA. Mice treated with phosphate-buffered saline were used as the negative control and mice treated with LPS only were used as the positive control. Data are expressed as the mean \pm standard deviation; ** P<0.01 vs. LPS group. BALF, bronchoalveolar lavage fluid; LPS, lipopolysaccharide; TNF- $\alpha$, tumour necrosis factor- $\alpha$; IL, interleukin; ctrl, negative control.

cell infiltration (Fig. 1B). However, lungs from the control and triptolide groups exhibited normal structures (Fig. 1A and C). These results indicate that triptolide improves the histopathological status of lungs in LPS-treated mice and inhibits the infiltration of the inflammatory cells into lung tissue.

Triptolide inhibits the expression of chemokines in the lung tissues of mice with ALI. Following LPS administration, the expression of the chemokines MIP-1 $\alpha$, MIP-1 $\beta$, RANTES, MCP-1 and IP-10, detected using qPCR, in lung tissue samples was markedly increased (Fig. 2). However, pretreatment with triptolide significantly reduced the LPS-induced expression of MIP-1 $\alpha$, MIP-1 $\beta$, RANTES, MCP-1 and IP-10 $(\mathrm{P}<0.05)$. No significant differences were observed in the expression of chemokines between the LPS + triptolide and control groups (Fig. 2).

Triptolide inhibits inflammatory cytokines in the BALF of mice with $A L I$. In order to elucidate the effect of triptolide on the mice with ALI, the mice were pretreated with different concentrations of triptolide and challenged with LPS. The concentrations of TNF- $\alpha$, IL- $1 \beta$, IL- 6 and IL- 8 in the BALF were then detected after $8 \mathrm{~h}$. It was found that the concentrations of the cytokines were significantly decreased in mice pretreated with triptolide in a dose-dependent manner (Fig. 3).

Triptolide efficiently decreases the MPO activity in lung tissues of mice injured by LPS. The MPO activity in lung tissues was significantly increased following treatment with LPS compared with the control tissues (Fig. 4). However, triptolide pretreatment markedly decreased the MPO activity compared with the LPS group $(\mathrm{P}<0.01)$.

Triptolide inhibits the lung edema of mice with ALI. Compared with the negative control group, the lung wet/dry weight ratios were significantly increased in the LPS group (Fig. 5); however, the wet/dry weight ratios of the lungs were significantly reduced by triptolide administration $(\mathrm{P}<0.01$, LPS + triptolide versus LPS groups). Thus, triptolide inhibits lung edema in mice with ALI.

Triptolide inhibits the NF- $\kappa B$ pathway in the A549 lung cell line. In order to elucidate the mechanism underlying the anti-inflammatory effects of triptolide, the A549 cell line 


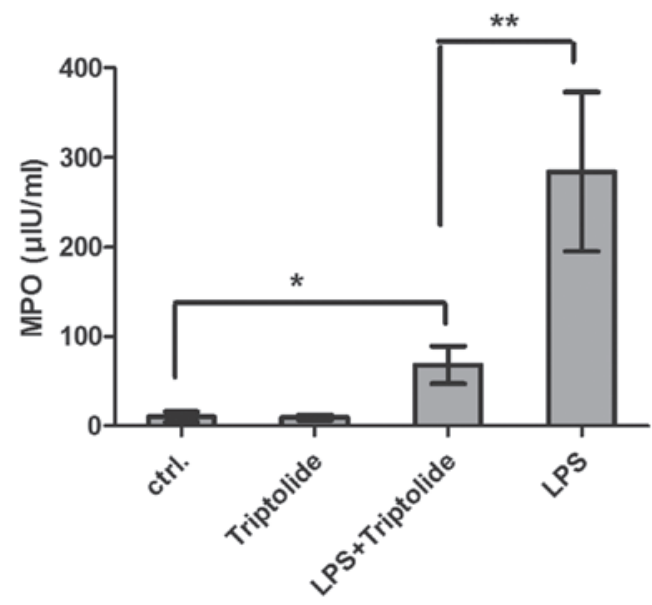

Figure 4. MPO activity. MPO activity was markedly reduced in the mice treated with triptolide and LPS compared with mice treated with LPS only. Data are expressed as the mean \pm standard deviation; ${ }^{*} \mathrm{P}<0.05$ vs. control and triptolide groups; ${ }^{* * *} \mathrm{P}<0.01$ vs. LPS group. MPO, myeloperoxidase; LPS, lipopolysaccharide; ctrl, negative control.

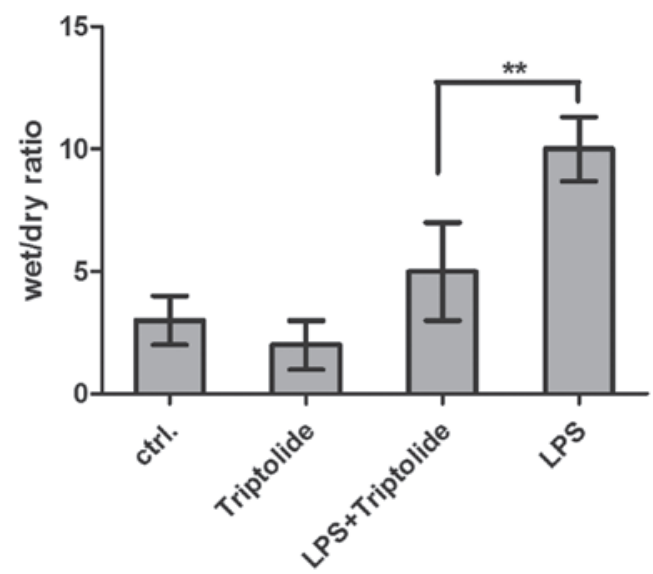

Figure 5. Wet/dry ratios in different groups. The wet/dry ratios were detected $8 \mathrm{~h}$ after LPS administration and reflected the lung edema of mice in the different treatment groups. Pretreatment with triptolide prior to LPS administration markedly decreased lung wet/dry ratios. Data are presented as the mean \pm standard deviation; ${ }^{* *} \mathrm{P}<0.01$ vs. LPS group. LPS, lipopolysaccharide; ctrl, negative control.

was used as a cell model. Following LPS administration, it was observed using western blot analysis that the expression levels of TLR4, p-I $\kappa \mathrm{B}-\alpha$ and p-NF- $\kappa \mathrm{B}$ p65 were significantly increased. However, $3 \mathrm{~h}$ after triptolide pretreatment, the LPS-induced expression of $\mathrm{p}-\mathrm{I} \kappa \mathrm{B}-\alpha$ and $\mathrm{p}-\mathrm{NF}-\kappa \mathrm{B}$ p 65 was significantly suppressed (Fig. 6).

\section{Discussion}

ALI is a type of diffuse alveolar injury and is a complex clinical syndrome that is initiated by injury to the lung $(24,25)$. In the present study, the effect of triptolide was investigated using a murine model of LPS-induced ALI. The activation and recruitment of neutrophils are considered to have an important role in the progression of ALI. IL-8 is a potent chemokine that attracts neutrophils to the inflammatory sites. In the present study it was demonstrated that, in the ALI model, chemokine
A

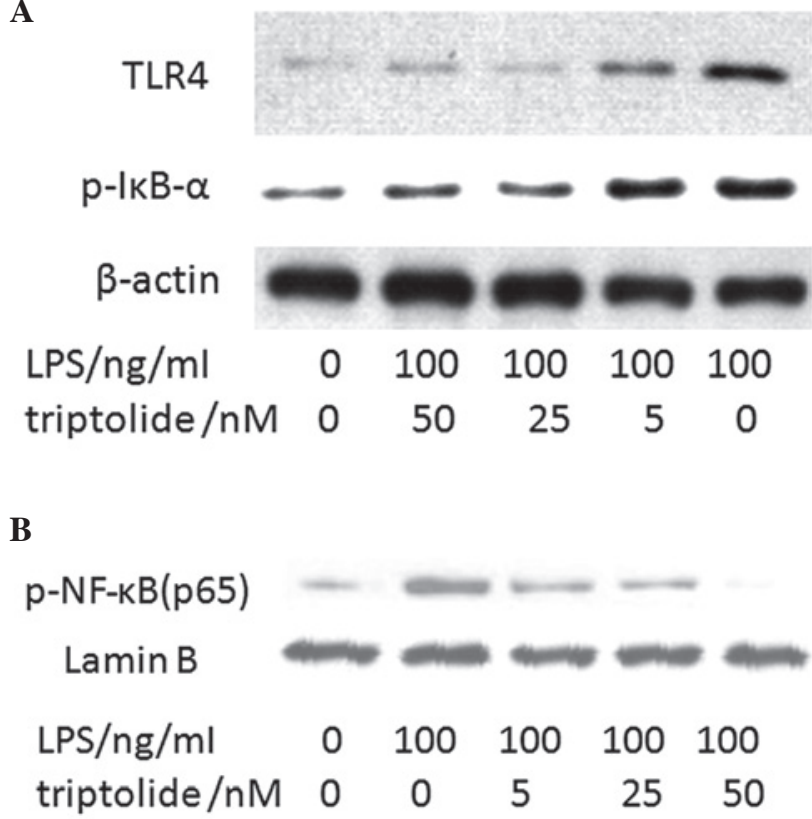

Figure 6. Effect of triptolide on the NF-кB pathway in mice treated with LPS in combination with triptolide. A549 cells were treated with $100 \mathrm{ng} / \mathrm{ml}$ LPS along with different concentrations of triptolide for $1 \mathrm{~h}$. Total cell lysates were analysed using western blotting. (A) Expression of TLR4 and p-IкB; (B) expression of $\mathrm{p}-\mathrm{NF}-\kappa \mathrm{B}$. A dose-dependent effect on gene expression may be observed. The results shown are representative of three independent experiments. NF- $\kappa \mathrm{B}$, nuclear factor $\kappa$-light-chain-enhancer of activated B cells; LPS, lipopolysaccharide; TLR4, Toll-like receptor 4; IKB- $\alpha$, nuclear factor of $\kappa$ light polypeptide gene enhancer in $B$ cells inhibitor- $\alpha$; $p-N F-\kappa B$,

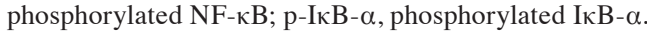

levels, including those of IL-8, IP-10, MIP-1 $\alpha$, MIP-1 $\beta$ and RANTES, were significantly increased in the BALF, and this was accompanied by the gathering of neutrophils, macrophages and monocytes to the inflammatory sites, which accelerated the progression of ALI. However, pretreatment with triptolide effectively inhibited the production of chemokines and, therefore, relieved the progression of ALI.

The local production of cytokines (TNF- $\alpha$, IL-1 and IL-6) by lung tissues in response to infectious or inflammatory stimuli may also attract immune cells, such as neutrophils, dendritic cells and monocytes, to the inflammatory sites $(26,27)$, leading to pulmonary injury. The cytokines also activate granulocytes, which cause acute neutrophilic inflammation and attract natural killer cells, monocytes and a variety of other immune cells $(28,29)$.

MPO, a major constituent of neutrophil cytoplasmic granules, and its activity in lung tissue are direct indicators of neutrophil sequestration in lung tissues (30). In the present study, the results demonstrated that MPO activity increased markedly in lung tissues following LPS treatment. Notably, pretreatment with triptolide significantly decreased the MPO activity. This finding was consistent with the results from the histopathological analysis, which demonstrated that pretreatment with triptolide markedly attenuated the neutrophil infiltration in lungs and maintained the normal structure and function of the lungs in murine models. It was also observed that there was a significant reduction in the pulmonary edema of the lungs following pretreatment with triptolide, as well as a reduction in the wet/dry ratios of lung tissues. 
The mechanism underlying the anti-inflammatory activity of triptolide was also investigated in the present study. It was indicated that triptolide may play a key role in the TLR4-NF- $\kappa$ B signalling pathway by inhibiting the expression of TLR4, decreasing the phosphorylation and degradation of $\mathrm{I} \kappa \mathrm{B}$ and inhibiting the consequent activation of NF- $\kappa \mathrm{B}(\mathrm{p} 65)$ to decrease the production of cytokines and alleviate the progression of ALI. Thus, the results from this study provide an insight for the development of triptolide-based therapies for the treatment of ALI. Furthermore, the findings may help to elucidate the mechanism and targets of triptolide in cells in anti-inflammatory responses.

\section{References}

1. Chang $\mathrm{CH}$, Kao $\mathrm{CH}$, Chio $\mathrm{CC}$, Lin $\mathrm{CH}$, Lin MT and Chang $\mathrm{CP}$ Attenuating heatstroke-induced acute lung inflammation, edema, and injury in rats by exercise preconditioning. J Trauma Acute Care Surg 74: 1052-1059, 2013.

2. Sharma S, Smith A, Kumar S, et al: Mechanisms of nitric oxide synthase uncoupling in endotoxin-induced acute lung injury: role of asymmetric dimethylarginine. Vascul Pharmacol 52: 182-190, 2010.

3. Fu PK, Yang CY, Tsai TH and Hsieh CL: Moutan cortex radicis improves lipopolysaccharide-induced acute lung injury in rats through anti-inflammation. Phytomedicine 19: 1206-1215, 2012.

4. Pati S, Gerber MH, Menge TD, et al: Bone marrow derived mesenchymal stem cells inhibit inflammation and preserve vascular endothelial integrity in the lungs after hemorrhagic shock. PLoS One 6: e25171, 2011.

5. Goodman RB, Pugin J, Lee JS and Matthay MA: Cytokine-mediated inflammation in acute lung injury. Cytokine Growth Factor Rev 14: 523-535, 2003.

6. Wen HL, Liang ZS, Zhang R and Yang K: Anti-inflammatory effects of triptolide improve left ventricular function in a rat model of diabetic cardiomyopathy. Cardiovasc Diabetol 12: 50, 2013.

7. Seki H, Tasaka S, Fukunaga K, et al: Effect of Toll-like receptor 4 inhibitor on LPS-induced lung injury. Inflamm Res 59: 837-845, 2010.

8. Jeyaseelan S, Chu HW, Young SK, Freeman MW and Worthen GS Distinct roles of pattern recognition receptors CD14 and Toll-like receptor 4 in acute lung injury. Infect Immun 73: 1754-1763, 2005

9. Kennedy M, Phelps D and Ingenito E: Mechanisms of surfactant dysfunction in early acute lung injury. Exp Lung Res 23: 171-189, 1997.

10. Ben DF, Yu XY, Ji GY, et al: TLR4 mediates lung injury and inflammation in intestinal ischemia-reperfusion. J Surg Res 174 326-333, 2012

11. Huang D, Fang F and Xu F: Hyperoxia-induced up-regulation of Toll-like receptors expression in alveolar epithelial cells. Zhongguo Wei Zhong Bing Ji Jiu Yi Xue 23: 645-649, 2011 (In Chinese).

12. Zhong WT, Wu YC, Xie XX, et al: Phillyrin attenuates LPS-induced pulmonary inflammation via suppression of MAPK and $\mathrm{NF}-\kappa \mathrm{B}$ activation in acute lung injury mice. Fitoterapia 90 : $132-139,2013$

13. Matta R, Wang X, Ge H, Ray W, Nelin LD and Liu Y: Triptolide induces anti-inflammatory cellular responses. Am J Transl Res 1: $267-282,2009$
14. Qiu D and Kao PN: Immunosuppressive and anti-inflammatory mechanisms of triptolide, the principal active diterpenoid from the Chinese medicinal herb Tripterygium wilfordii Hook. f. Drugs R D 4: 1-18, 2003.

15. Hoyle GW, Hoyle CI, Chen J, Chang W, Williams RW and Rando RJ: Identification of triptolide, a natural diterpenoid compound, as an inhibitor of lung inflammation. Am J Physiol Lung Cell Mol Physiol 298: L830-L836, 2010.

16. Lee HF, Lee TS and Kou YR: Anti-inflammatory and neuroprotective effects of triptolide on traumatic brain injury in rats. Respir Physiol Neurobiol 182: 1-8, 2012.

17. Klawitter M, Quero L, Klasen J, et al: Triptolide exhibits anti-inflammatory, anti-catabolic as well as anabolic effects and suppresses TLR expression and MAPK activity in IL-1 $\beta$ treated human intervertebral disc cells. Eur Spine J 21 (Suppl 6): S850-S859, 2012.

18. Mei Z, Li X, Wu Q, Hu S and Yang X: The research on the anti-inflammatory activity and hepatotoxicity of triptolide-loaded solid lipid nanoparticle. Pharmacol Res 51: 345-351, 2005.

19. Jeyaseelan S, Chu HW, Young SK and Worthen GS: Transcriptional profiling of lipopolysaccharide-induced acute lung injury. Infect Immun 72: 7247-7256, 2004.

20. Guo Z, Li Q, Han Y, Liang Y, Xu Z and Ren T: Prevention of LPS-induced acute lung injury in mice by progranulin. Mediators Inflamm 2012: 540794, 2012.

21. Fujino N, Kubo H, Suzuki T, et al: Administration of a specific inhibitor of neutrophil elastase attenuates pulmonary fibrosis after acute lung injury in mice. Exp Lung Res 38: 28-36, 2012.

22. Lewis CW, Taylor RG, Kubara PM, Marshall K, Meijer L and Golsteyn RM: A western blot assay to measure cyclin dependent kinase activity in cells or in vitro without the use of radioisotopes. FEBS Lett 587: 3089-3095, 2013.

23. Vallejo-Illarramendi A, Marciano DK and Reichardt LF: A nove method that improves sensitivity of protein detection in PAGE and Western blot. Electrophoresis 34: 1148-1150, 2013.

24. Lim YS, Chung MH, Park SH, et al: Acute and repeated inhalation lung injury by 3 -methoxybutyl chloroformate in rats: CT-pathologic correlation. Eur J Radiol 62: 227-234, 2007.

25. Parsons PE, Eisner MD, Thompson BT, et al; NHLBI Acute Respiratory Distress Syndrome Clinical Trials Network: Lower tidal volume ventilation and plasma cytokine markers of inflammation in patients with acute lung injury. Crit Care Med 33: 1-6; discussion 230-232, 2005 .

26. Trevejo JM, Marino MW, Philpott N, et al: TNF-alpha-dependent maturation of local dendritic cells is critical for activating the adaptive immune response to virus infection. Proc Natl Acad Sci USA 98: 12162-12167, 2001.

27. Ning J, Liu QF, Luo XD, Fan ZP and Zhang Y: Effect and mechanism of acute graft versus host disease on early diffuse murine lung injury following allogeneic stem cell transplantation. Sci China C Life Sci 52: 1016-1022, 2009.

28. Wu Y, Wan T, Zhou X, et al: Hsp70-like protein 1 fusion protein enhances induction of carcinoembryonic antigen-specific $\mathrm{CD} 8^{+}$CTL response by dendritic cell vaccine. Cancer Res 65: 4947-4954, 2005.

29. Zhang N, Wang Z, Tang X, et al: Type $1 \mathrm{~T}$-cell responses in chlamydial lung infections are associated with local MIP-1alpha response. Cell Mol Immunol 7: 355-360, 2010.

30. Peng XM, Xie JH, Shuai B, et al: Inhalation of aerosolized perfluorocarbon combined with tetramethylpyrazine ameliorates hemodynamics and pulmonary histopathology in a porcine model of acute lung injury. Nan Fang Yi Ke Da Xue Xue Bao 31: 1382-1386, 2011 (In Chinese). 